\title{
Experiencia de creación del MOOC "Música para el siglo XXI: aportaciones del software libre a la educación musical”
}

\author{
Chao-Fernández, Rocío; Felpeto-Guerrero, Abraham; Vázquez-Sánchez, Rubén
}

Grupo Innovación educativa IMETIC, Facultad de Ciencias de la Educación,

Universidade da Coruña.

\section{RESUMEN}

Con motivo de la I convocatoria para la elaboración de MOOCS de la Universidade da Coruña y dada la ausencia de cursos específicos relacionados con música y TIC dentro de las plataformas más importantes de MOOCS, desde el grupo de innovación educativa metodológica a través de las TIC (IMETIC), y en colaboración con la Universidad de Cádiz, se decide elaborar un Mooc centrado en el software libre y la educación musical. En la presente comunicación abordamos la experiencia de preparación de este MOOC centrándonos en la explicación de cómo ha sido el proceso de elaboración del mismo, desde la planificación y selección de los módulos que componen el curso hasta el proceso de decisión de los tipos de material audiovisual necesario para el mismo, explicando cómo hemos realizado las grabaciones, y los diferentes materiales de apoyo. Una vez generados todos los materiales didácticos, se expone el flujo de trabajo necesario para la publicación de dichos contenidos en la plataforma Mooc escogida a tal efecto, en este caso MiríadaX.

PALABRAS CLAVE: Mooc, educación musical, MiríadaX, software libre, proceso de creación 


\section{CITA RECOMENDADA:}

Chao-Fernández, R.; Felpeto Guerrero, A. ; Vázquez-Sánchez, R.(2019): Experiencia de creación del MOOC "Música para el siglo XXI: aportaciones del software libre a la educación musical". En De la Torre Fernández, E. (ed.) (2019). Contextos universitarios transformadores: construíndo espazos de aprendizaxe. III Xornadas de Innovación Docente. Cufie. Universidade da Coruña. A Coruña (pág. 25-40).

DOI capítulo: https://doi.org/10.17979/spudc.9788497497121.025

DOl libro: https://doi.org/10.17979/spudc.9788497497121

\section{ABSTRACT}

On the ocassion of the I call for the development of MOOC courses organized by University of A Coruña and given the absence of specific courses related with music and ICT withing the most important MOOC platforms, teachers from IMETIC group in collaboration with University of Cadiz decided to develop a MOOC course focused on open-source and music education. Through the present communication we address the experience of the creation of this $\mathrm{MOOC}$, focusing on the explanation about how the course was prepaired, from the planning and selection of the modules that make up the course up to the decision about the kind of audiovisal materials neccesary for it, going through the explanation about how we made the recordings as well as other support materials. Once all the didactic materials have been generated, we explain how was the workflow followed in order to publish all the contents in the MOOC platform chosen for this purpose, in this case MiríadaX.

KEY WORDS: Mooc, music education, MiríadaX, open-source, development process 


\section{INTRODUCCIÓN}

¿Qué es un M00C? Podríamos extraer una definición derivada del propio significado de su acrónimo, "Massive Open On-line Course"; o su equivalente en castellano COMA, "Curso Online Masivo y Abierto". Se trata de una tipología de curso que se origina en 2008 a raíz de un curso online sobre conectivismo realizado por Siemens y Downes (Vázquez Cano et al., 2013). Entre las características principales destacan su semejanza en cierta medida con un aula, que tienen fechas de inicio y final definidas de antemano, son online y gratuitos a través de internet de forma que cualquier persona puede acceder a ellos, y cuentan con una serie de mecanismos que permiten realizar la evaluación del alumnado (Cabero-Almenara et al., 2014). Tras detectar que en las diferentes plataformas MOOC más importantes en lengua española no era posible localizar cursos sobre música destinados específicamente a docentes ${ }^{i}$ - tanto futuros como en activo - unido a nuestra creencia de que la faceta artístico-musical ha de promoverse a través de metodologías ágiles de e-learning, el grupo de investigación IMETIC, integrado por docentes de las Universidades de Cádiz y de A Coruña decide presentar esta propuesta de curso MOOC a la I convocatoria para la elaboración de M00C's de la Universidade de A Coruña.

Una vez elaborada, presentada y aprobada, llegaba el momento de llevarla a cabo, y es aquí donde surgen los primeros problemas a raíz de la inexperiencia del equipo docente dentro del ámbito de este tipo de cursosii, aflorando preguntas como ien qué plataforma y qué requisitos debemos satisfacer para publicar nuestro curso? ¿qué tipos de materiales didácticos debemos crear y cómo los creamos? ¿quién nos puede ayudar con todo el proceso? ¿cuál es el profesorado ideal para el curso?, entre otras muchas cuestiones. 


\section{LA EXPERIENCIA}

Dado que son muchas las cuestiones a tener en cuenta a la hora de crear un curso MOOC, trataremos de desengranar los aspectos principales en base a la propia experiencia vivida por este equipo. Para tratar de ofrecer una narración lo más ordenada posible dividiremos el relato de la experiencia en los apartados que mayor relevancia han tenido.

\subsection{PROFESORADO Y PERSONAL DE APOYO}

Se ha requerido una amplia variedad de profesionales de distintos ámbitos para poder llevar a cabo este MOOC. A priori pudiera parecer que el personal fundamental es el profesorado, la cara visible del curso de cara al alumnado, pero nada más lejos de la realidad, dado que existen numerosos aspectos a tener en cuenta además de la propia tutorización del alumnado. En el caso concreto que nos atañe ha sido partícipe el siguiente equipo:

- Profesorado de las universidades de Cádiz y A Coruña: Sobre el que recae el grueso de las tareas. Es imprescindible que sea profesorado todoterreno dado que, además de su conocimiento sobre educación y tecnología musical, no deberían tener miedo a ponerse frente a una cámara ni a quedar grabados en plataformas abiertas. Además, como se explicará más adelante, deben tener conocimientos en elaboración y edición de vídeos didácticos y poseer cualidades dinamizadoras para poder gestionar a distancia el grupo de alumnos matriculado en el curso.

- Técnicos de teleformación: Su función principal está en colaborar en la toma de decisiones sobre cómo estructurar el curso y los diferentes módulos formativos, además de hacer de intermediarios entre el personal de soporte de la plataforma MOOC escogida y el profesorado del curso.

- Personal técnico de estudio de grabación: Imprescindible para organizar los setup de grabación en estudio, así como para velar por el correcto desarrollo de las sesiones de grabación. 
- Técnicos informáticos: Su función es la de instruir al profesorado en el uso del software de grabación y edición. Además, colaboran en el maquetado del curso dentro de la plataforma, en el post-procesado y alojamiento de los vídeos en la nube.

- Diseñador gráfico: Su labor consiste en crear el apartado visual del curso, como pueden ser infografías, plantillas, identidad visual, cabeceras de los vídeos, entre otros.

- Alumnado en prácticas de la especialidad de imagen y sonido: Su inestimable ayuda ha servido para revisar que todas las grabaciones realizadas tenían un correcto nivel de volumen y nitidez de imagen además de realizar la introducción de infografías y textos durante la post-producción.

\subsection{CONTENIDOS}

Una vez que se cuenta con el personal necesario, llega el momento de pensar en los contenidos. Independientemente de la temática, los hemos estructurado de tal forma que trataran de paliar, en la medida de lo posible, el fenómeno conocido como embudo de participación (Clow, 2013) y evitar la pérdida de participantes en el desarrollo del curso, que normalmente se cifra en el $90 \%$ del alumnado. Para ello se toman tres medidas.

En primer lugar, todos los contenidos de aprendizaje deberían ser independientes entre sí, esto es, para poder acceder a los contenidos de un determinado módulo, no fuera necesario haber adquirido los contenidos desarrollados en alguno de los otros, quedando establecida de esta forma una jerarquía horizontal entre módulos.

En segundo lugar, los contenidos deberían permitir diferentes ritmos de aprendizaje. Si por algo se caracteriza un MOOC es por la gran diversidad de individuos que lo cursan, creando una masa de participantes totalmente heterogénea en cuanto a lo que ritmo se refiere. Muchos de los MOOC que habíamos revisado antes de crear el nuestro, ofrecían una temporalización de módulos estricta, viéndose forzado muchas veces su alumnado a acelerar o disminuir su velocidad de aprendizaje en función de las fechas de inicio y finalización de cada módulo. En 
nuestro caso decidimos que se abrirían y cerrarían todos los módulos al mismo tiempo, coincidiendo con las fechas de inicio y finalización del curso con la única salvedad de la tarea obligatoria P2P. Con esta configuración se ofrece al alumnado la capacidad de poder distribuir de forma más eficiente su tiempo de aprendizaje pudiendo acelerar o ralentizar el ritmo en aquellos módulos o contenidos que así lo precisen, sin verse condicionados por un calendario cerrado.

En tercer lugar, los contenidos deberían adaptarse a diferentes niveles de conocimiento. Aunque el curso está destinado fundamentalmente a futuros maestros y a profesores en activo, esto no quiere decir que no haya otro tipo de perfil de alumno interesado. Por este motivo, dentro de una misma lección incluyen contenidos de desarrollo básicos, idóneos para todos los niveles de aprendizaje y, para aquel alumnado más avanzado o con ávida curiosidad por aprender más sobre el tema, se incluye una sección no obligatoria titulada "para saber más", con contenidos que permiten un desarrollo más completo de cada uno de los conceptos presentados.

Así, teniendo en cuenta las tres premisas anteriores, el curso quedaría secuenciado de la siguiente forma:

- Módulo introductorio: También llamado módulo 0, requisito de obligada introducción por parte de la plataforma utilizada, en el que simplemente añadimos un mensaje de bienvenida al curso y una pequeña encuesta para conocer por lo general cuál es el perfil del alumnado.

- Módulos temáticos: Cuatro en su total, cada uno de ellos dedicado al uso de una herramienta de software libre diferente. Cada uno de ellos incluye un pequeño cuestionario de autoevaluación.

- Módulo de evaluación: Cuyo contenido único es el desarrollo de una actividad P2P centrada en la elaboración de un paisaje sonoro imaginario y original hecho con Audacity. 
En relación con los módulos temáticos, el primero de ellos titulado Competencia Mediática, TIC Y Software Libre En E. Musical podría considerarse como un punto de partida o visión conceptual del curso, abordando el concepto general de competencia mediática, la incorporación de las TIC al campo musical y los beneficios de la utilización del software libre en la enseñanza de la música. Los siguientes módulos están dedicados al uso de las herramientas de software libre, perteneciendo cada una de ellas a un ámbito diferente, pero que en su conjunto forman parte de los pilares fundamentales en los que se centra la educación musical. Así, el módulo 2 titulado Lenguaje Musical y Desarrollo Auditivo: LenMus; está dedicado al entrenamiento progresivo del oído musical además de poder trabajar conceptos musicales teóricos. El módulo 3 titulado Crea y edita tus propias partituras: MuseScore; se centra en el proceso de aprendizaje de la escritura musical a través de un software de edición de partituras y finalmente el módulo 4 titulado Creación y edición musical: Audacity; se centra en la creatividad musical a través del uso de una herramienta de grabación y edición de sonidos multipista.

A efectos de certificación, superar los módulos introductorio y temáticos conducirían a la obtención del certificado de participación del curso, otorgándose el certificado de superación del $100 \%$ del curso únicamente a aquel alumnado que además de los anteriores, haya cursado y superado el módulo de evaluación.

\subsection{MATERIALES DIDÁCTICOS}

Una vez conocidos los contenidos a desarrollar hay que plasmarlos en los diferentes materiales didácticos. Al igual que en prácticamente la mayoría de MOOC existentes, el material didáctico por excelencia es el vídeo bajo demanda, que posibilita al alumnado seguir las diferentes lecciones $\mathrm{y} / 0$ explicaciones del profesorado en cualquier momento y desde cualquier tipo de dispositivo con una conexión a internet. Además, permiten una mayor versatilidad, de forma que el alumnado lo pueda pausar y reanudar mientras maneja las diferentes herramientas de software libre, 0 avanzar rápidamente hasta aquel punto que 
consideren sin tener que hacer una visualización completa del mismo, en especial cuando se trata de repasar los contenidos aprendidos anteriormente.

En este curso, dependiendo del propósito a alcanzar se utilizan dos tipos diferentes de vídeo: en primer lugar, los grabados en estudio, que son los utilizados a modo introductorio de cada uno de los módulos temáticos. Permiten al alumnado saber de forma rápida cuáles son los principales aspectos a tratar en cada uno de los mismos, así como la forma en la que se va a trabajar 0 abordar los diferentes contenidos. Se decide grabar en estudio estos vídeos por su importancia como punto de partida hacia cada módulo temático ya que, si el vídeo no atrae, la pérdida de alumnos posiblemente sería más acusada. Estos vídeos constan de la imagen del profesor a pantalla completa junto con una serie de textos o infografías que van acompañando su discurso. En segundo lugar, se utilizan vídeos en screencast o de grabación de pantalla a modo de videotutoriales en los que el profesorado de cada módulo muestra los contenidos en las lecciones. Se decide grabar las lecciones de este modo por la mayor facilidad que conlleva planear todo el proceso de grabación, no teniendo que depender de la disponibilidad del estudio ni del equipo técnico, primando además la visualización del uso de cada una de las aplicaciones. En este caso, aunque no se vea el profesor, los vídeos en screencast incluyen su voz en off explicando cada uno de los pasos dados.

A la hora de afrontar las grabaciones se deben tener en cuenta una serie de aspectos que condicionarán su buen resultado:

- Guión preparado éxito asegurado: Tanto los vídeos de estudio como los vídeos en screencast han de ser planificados con anterioridad a su grabación. Para ello se hará uso del storyboard o guión, en el cual se detallará tanto el discurso como las acciones o pasos a dar en la aplicación, esto último en caso de los screencast.

- Ensayar es anticipar: Antes de acudir al estudio o de hacer la grabación screencast es de vital importancia ensayar el guión, tanto para agilizar el proceso de grabación como para afrontar la grabación con mayor seguridad y aplomo. 
- Dejar el miedo en casa: Si hay algo que es cierto es que la cámara impone y huele el miedo. Especialmente al abordar las grabaciones en estudio, hay que intentar conseguir un ambiente de rodaje lo más distendido posible, evitando poses tensas y titubeos en la voz. Una ayuda es colocar encima del objetivo de la cámara un objeto gracioso (muñeco, post it con una cara sonriente, etc) que ayude a cortar el hielo. Tener un bolígrafo o tótem en las manos también permitirá ganar confianza, pero ha de evitarse moverlo en exceso.

- Silencio, ise rueda!: En el estudio no habrá mayor problema si se encuentra bien aislado, pero al grabar los screencast en casa o en el despacho hay que procurar que no haya ningún ruido que pueda arruinar el trabajo. Por ello se recomienda avisar a la gente que pueda interrumpirnos durante la grabación. Es útil colocar un cartel en la puerta haciendo saber que nadie puede entrar en ese momento. Tanto para las grabaciones en estudio como para los screencast, es importante dejar el teléfono móvil desconectado para que no interfiera en la microfonía.

- Cuidar la vestimenta: Para grabar en estudio, en especial si se utiliza la técnica chroma key 0 fondo verde, hay que procurar evitar prendas con franjas pequeñas 0 con colores que puedan interferir con el fondo del estudio, normalmente verde.

Además de los factores anteriores, que están relacionados con el proceso de grabación también ha de cuidarse el resultado final de dichas grabaciones de tal forma que:

- El vídeo ha de tener buena calidad de imagen: Se grabará siempre que se pueda con cámara full HD y con el escritorio en resolución HD.

- Los vídeos han de ser vistosos evitando la monotonía: Estamos en el ámbito de la educación no formal, por tanto, debemos encontrar el término medio entre el formalismo y la vulgaridad a la hora de plantear el set up "iide nuestros vídeos y el lenguaje a utilizar. 
- Es de vital importancia que la duración de todos los vídeos sea lo más corta posible. Es preferible tener las lecciones divididas en varios vídeos cortos, que en pocos y largos, ya que son más fáciles de seguir y evitan el efecto de desconexión. Estudios realizados sobre plataformas $\mathrm{MOOC}$ recomiendan que la duración ideal es de 6 minutos (Guo, 2013).

- Comprobar todo el material: Especialmente al acudir al estudio, al finalizar las grabaciones se debe comprobar que se lleven a postproducción las tomas correctas y que la calidad de imagen sea la adecuada a fin de evitar repeticiones.

Además de los vídeos de presentación y de contenidos didácticos de cada lección, se decide incluir otro tipo de material didáctico cuya finalidad es facilitar los diferentes estilos de aprendizaje del alumnado ${ }^{\text {iv }}$. Por ello se incluye en cada lección uno 0 varios documentos de texto con los mismos contenidos tratados en los vídeos, apoyados en infografías e imágenes, donde además se resaltan todos aquellos aspectos más importantes de cada lección. Asimismo, se adjuntan las transcripciones de los vídeos y una serie de recursos extra como bibliografía o enlaces relacionados.

Todos los materiales didácticos elaborados para el curso se distribuyen bajo licencia Creative Commons 4.0 Reconocimiento-NoComercial-Compartirlgual

\subsection{COMPLEMENTOS DIDÁCTICOS}

Además de los materiales didácticos propiamente dichos, se ha querido facilitar la interacción social entre el alumnado de forma que el aprendizaje no sea simplemente vertical, sino que también se pueda desarrollar horizontalmente entre ellos. Así, se dinamizó el curso incluyendo por una parte, un blog en el que diariamente el equipo docente publicaba entradas de actualidad relacionadas con el contenido del curso, intentando suscitar la curiosidad del alumnado para continuar aprendiendo y, por otra parte creando foros temáticos para promover la interacción anteriormente comentada. Por cada uno de los módulos se abrió un foro, que 
además de servir de lugar donde plantear dudas relacionadas con los contenidos de aprendizaje, el propio alumnado pudiera plantear cuestiones relacionadas con el módulo pero no tratadas en el mismo, de forma que se fomentara una actividad de descubrimiento de nuevos contenidos. Además, se incluyen dos foros generales de presentaciones y offtopic 0 cafetería, donde se pueden plantear temas o cuestiones fuera del ámbito del curso.

\subsection{LA PLATAFORMA}

Coursera, EdX, Udacity, Canvas... existe una gran cantidad de plataformas en las que publicar cursos MOOC. En este caso se decide publicar el curso a través de la plataforma MiríadaX, fundamentalmente por 3 motivos: es la que mayor comunidad de alumnado hispanohablante posee, permitiéndonos llegar a un mayor número potencial de alumnos que utilizando plataformas mayores cuya lengua oficial es el inglés. Resulta muy sencillo navegar por ella y tiene una estructura limpia comparando con otras plataformas que el equipo había utilizado y, por último, posee personal de soporte técnico en español y de fácil acceso en caso de tener que resolver incidencias. Por el contrario, ofrece una variedad de recursos limitada en cuanto a los tipos de materiales didácticos que se pueden utilizarv y que, dependiendo de la hora del día de acceso, es posible que la plataforma experimente velocidades de carga lentas y errores debido al elevado tráfico web ${ }^{\text {vi }}$.

De cara a la creación de curso en MiríadaX, es requisito obligatorio satisfacer los aspectos mostrados en la figura 1. 


\begin{tabular}{|c|c|}
\hline & REQUISITOS OBLIGATORIOS DE MIRIADA X \\
\hline \multicolumn{2}{|c|}{ Duración comprendida entre 4 semanas y 12 semanas } \\
\hline \multicolumn{2}{|c|}{$\begin{array}{l}\text { Vídeo de presentación, publicado en Youtube y de máximo } 5 \text { minutos de } \\
\text { duración }\end{array}$} \\
\hline \multicolumn{2}{|c|}{ Estructura organizada por módulos } \\
\hline \multirow{3}{*}{$\begin{array}{l}\text { Módulo } 0 \\
\text { introductorio } \\
\text { con: }\end{array}$} & Video descriptivo del curso \\
\hline & Texto con breve descripción \\
\hline & $\begin{array}{l}\text { Evaluación inicial (Ej. evaluación de conocimientos previos, } \\
\text { encuesta demográfica, encuesta de expectativas, etc.) }\end{array}$ \\
\hline \multirow{3}{*}{$\begin{array}{l}\text { El resto de } \\
\text { módulos deben } \\
\text { incluir: }\end{array}$} & $\begin{array}{l}\text { Material audiovisual (Videos narrados por los docentes, } \\
\text { subtitulados y alojados en Youtube) }\end{array}$ \\
\hline & $\begin{array}{l}\text { Material complementario (Documentación de apoyo como } \\
\text { enlaces externos, lecturas, etc.) }\end{array}$ \\
\hline & Sistema de evaluación (Cuestionarios y Actividades P2P) \\
\hline \multicolumn{2}{|c|}{ Creación de un Foro } \\
\hline \multicolumn{2}{|c|}{ Materiales publicados bajo licencia Creative Commons } \\
\hline
\end{tabular}

Figura 1. Requisitos obligatorios de MiríadaX (Fuente: Universidad Politécnica de Madrid).

En cuanto al flujo de trabajo para alojar todo el material y maquetar el curso dentro de MiríadaX, se han seguido de forma resumida los siguientes pasos:

- Crear la estructura básica del curso: Engloba la creación de los diferentes módulos y dentro de ellos sus correspondientes lecciones. En cada lección será necesario enlazar los vídeos, crear y/o maquetar las entradas de texto html que se mostrarán junto a los vídeos, subir los documentos y materiales didácticos y establecer los ajustes tales como obligatoriedad de visualizar la lección y fecha de inicio y fin de cada módulo.

- Añadir las actividades: Una vez creada la estructura básica del curso y sus lecciones, es el momento de añadir las actividades de evaluación, siendo para el caso de los módulos temáticos cuestionarios de tipo autoevaluación sin límite de intentos ni fecha límite, y en el caso de la tarea P2P estableciéndola como obligatoria. 
- Creación de los foros, blog y mantenimiento/dinamización: Diariamente se establece un profesor encargado de publicar una entrada en el blog y se revisan una vez al día los mensajes del foro a fin de responder a cualquier duda del alumnado.

Una vez creada toda la estructura del curso, se aconseja encarecidamente hacer capturas de pantalla de cada módulo/lección para tener como soporte sobre cómo se han dispuesto y configurado todos los elementos. Además, se guardarán todos los textos en html y enlaces a vídeos ante el hipotético caso de que desaparezcan o se desconfiguren. De este modo, se tendrá una copia de seguridad para consultar en caso de tener que volver a restablecer la configuración inicial.

\section{RESULTADOS}

Como resultado de la experiencia de elaboración de este primer MOOC no podríamos estar más contentos de los logros alcanzados. Se ha llegado a la cifra de 1307 alumnos matriculados, que una vez cerrado el curso continúa incrementándose hasta los 1735 a la hora de cerrar este escrito. El sentimiento general de todo el personal implicado es de total satisfacción, en gran medida gracias al gran número de comentarios positivos que se ha recibido por parte del alumnado a través de los foros y redes sociales. Además, el curso ha resultado agraciado como finalista ${ }^{\text {vii }}$ al premio Innovación Educativa MiríadaX, entre 88 candidaturas de 44 universidades y 8 países diferentes.

\section{REFERENCIAS}

Cabero-Almenara, J., Llorente-Cejudo, M. C. \& Vázquez Martínez, A. I. (2014). Las tipologías de M00C: su diseño e implicaciones educativas. Profesorado, Revista de currículum y formación del profesorado, vol 18(1), pp. 17-26 
Clow, D. (2013). MOOCs and the funnel of participation. En D. Suthers, K. Verbert, E. Duval \& X. Ochoa (Eds.) Third International Conference on Learning Analytics and Knowledge (pp. 185189). New York: ACM.

Guo, P. (2013). Optimal Video Length for Student Engagement. Disponible en https://blog.edx.org/optimal-video-length-student-engagement

McAuley, A., Stewart, B., Siemens, G. \& Cormier, D. (2010). The MOOC model for digital practice. Island: University of Prince Edward Island. Disponible en: https://oerknowledgecloud.org/sites/oerknowledgecloud.org/

Sánchez-Vera, M. M., \& Prendes-Espinosa, M. P. (2013). La participación del alumnado en los cursos masivos (MOOC). Il Congreso Internacional de Innovación Docente. Disponible en http://digitum.um.es/xmlui/bitstream/10201/39138/1/C156_mooc.pdf

Universidad Politécnica de Madrid. Mooc: Guía para el docente. Madrid: Vicerrectorado de Servicios Tecnológicos - Gabinete de Tele-Educación. Disponible en http://serviciosgate.upm.es/docs/asesoramiento/Guia\%20M00C\%20para\%20el\%20profesor.p df

Vázquez Cano, E.; López Meneses, E.; Sarasola Sánchez-Serrano J. L. (2013). La expansión del conocimiento en abierto: Ios MOOC. Barcelona: Octaedro-ICE-UB.

\footnotetext{
' Es posible encontrar algunos cursos gratuitos sobre música por ejemplo, referidos a producción musical profesional, pero ninguno referido a la educación musical.

ii Aunque si bien tenía experiencia en el campo de plataformas de e-learning con Moodle o Chamilo entre otros.

iii Por set up se refiere a la composición del vídeo, es decir, la imagen, lugar o vídeo que aparecerá de fondo sustituyendo al chroma key, la organización espacial de los diferentes elementos a visualizar en pantalla, la gama cromática de los elementos, etc.
} 
iv Es probable que aunque la mayoría del alumnado prefiera utilizar los vídeos para consultar los contenidos, otra parte prefiera descargar un documento, imprimirlo y hacer anotaciones sobre el mismo.

${ }^{\vee}$ En nuestro caso, para esta primera edición del curso, era suficiente.

vi Esto ocurre especialmente durante la noche española, que es cuando se conecta un mayor número de usuarios desde Hispanoamérica.

vii $A$ falta del fallo final del jurado. 
